\title{
Chest magnetic resonance imaging for pneumonia diagnosis in outpatients with lower respiratory tract infection
}

\author{
Hannu Syrjala ${ }^{1,5}$, Markku Broas $^{4,5}$, Pasi Ohtonen ${ }^{3}$, Airi Jartti ${ }^{2}$ and Eija Pääkkö ${ }^{2}$ \\ Affiliations: 'Dept of Infection Control, Oulu University Hospital, Oulu, Finland. ${ }^{2}$ Dept of Diagnostic Radiology, \\ Oulu University Hospital, Oulu, Finland. ${ }^{3}$ Dept of Anesthesiology, Oulu University Hospital, Oulu, Finland. ${ }^{4}$ Dept of \\ Infection Control, Lapland Central Hospital, Rovaniemi, Finland. ${ }^{5}$ These authors contributed equally to this work.
}

Correspondence: Hannu Syrjala, Dept of Infection Control, Oulu University Hospital, Box 21, 90029 OYS, Finland. E-mail: hannu.syrjalaAppshp.fi

@ERSpublications

Chest MRI identified cases of pneumonia among 77 outpatients more often than chest radiography: 30 versus 23, $\mathrm{p}=\mathbf{0 . 0 4}$ http://ow.ly/Vj5T304FWkl

Cite this article as: Syrjala $\mathrm{H}$, Broas $\mathrm{M}$, Ohtonen $\mathrm{P}$, et al. Chest magnetic resonance imaging for pneumonia diagnosis in outpatients with lower respiratory tract infection. Eur Respir J 2017; 49: 1601303 [https://oi.org/ 10.1183/13993003.01303-2016].

ABSTRACT We assessed whether magnetic resonance imaging (MRI) is applicable for diagnosing pneumonia among adult outpatients with lower respiratory tract infection.

Patients were recruited prospectively when they had symptoms for $\leqslant 7$ days and fever $\geqslant 37.8^{\circ} \mathrm{C}$. The patients underwent high-resolution computed tomography (HRCT), MRI and chest radiography, which were repeated 1 month later if necessary, to verify pneumonia. The duration of MRI examination was 3-4 min.

Among 77 patients, HRCT, MRI and radiography identified 32 (41.6\%), 30 (39.0\%) and 23 (29.9\%) cases of pneumonia, respectively $(\mathrm{p}=0.001)$. MRI missed two HRCT-identified pneumonia cases due to motion artefacts. Chest radiography resulted in four false-positive pneumonia findings and MRI resulted in none. When HRCT was used as a reference, MRI had a sensitivity of 0.938 (95\% CI 0.799-0.983) and specificity of 0.978 (95\% CI $0.884-0.996$ ) for the diagnosis of pneumonia, whereas the sensitivity and specificity for chest radiography were 0.719 (95\% CI 0.546-0.844) and 0.911 (95\% CI 0.793-0.965), respectively.

MRI is an accurate, fast and effective method for diagnosing outpatient pneumonia, with better accuracy compared with chest radiography and almost similar accuracy compared with HRCT.

Received: July 012016 | Accepted after revision: Sept 262016

Support statement: H. Syrjala was supported by a Terttu grant of Healthcare foundation of Northern Finland. M. Broas was supported by an EVO grant from Lapland Central Hospital.

Conflict of interest: None declared.

Copyright @ERS 2017 


\section{Introduction}

Chest radiography is the standard method for verifying the diagnosis of community-acquired pneumonia (CAP) [1-3]. However, there are situations in which the use of ionising radiation should be avoided, such as in paediatric, young or pregnant patients. Magnetic resonance imaging (MRI) has been used successfully in paediatric pulmonary diseases [4-7], including pneumonia, as well as in detecting pulmonary abnormalities in immunocompromised patients [8-10]. MRI of the lung is challenging due to sparse soft tissue structures and low proton density for signal production [11]. Moreover, multiple interfaces between air and soft tissue generate susceptibility artefacts and fast signal decay. The motion of respiratory, cardiac and vascular systems may also cause artefacts. Despite these challenges, MRI of the lung is a promising diagnostic tool, and it has been extensively surveyed in four recent reviews [11-14] that presented excellent soft tissue contrast and functional information with this modality. MRI has been demonstrated to be as effective as high-resolution computed tomography (HRCT) in assessing the character of lung abnormalities among children and young adults with non-cystic fibrosis lung disease [15].

The aim of the present study was to estimate the diagnostic accuracy of chest MRI for identifying pneumonia among adult ambulatory patients with lower respiratory tract infection (LRTI). MRI results were compared with chest radiography findings obtained at the same time. We used HRCT as a reference [16] because it is superior to chest radiography in demonstrating pulmonary opacities in CAP patients [17-19].

\section{Methods}

The prospectively recruited study population comprised LRTI patients aged $\geqslant 18$ years who consulted outpatient clinics because of respiratory symptoms lasting $\leqslant 7$ days with fever $\geqslant 37.8^{\circ} \mathrm{C}$ and who had not received antibiotics for their ongoing respiratory infection. All participants volunteered and provided written informed consent. Patients with HIV infection and those hospitalised during the preceding 30 days or living in a nursing home were excluded. The study protocol was approved by the Ethics Review Committee of Lapland Central Hospital, Rovaniemi, Finland. The study (EudraCT 2012-002093-31) was conducted in accordance with the Helsinki Declaration of 2013. Patients were seen by a physician specialised in infectious diseases (M.B.) at the emergency department of Lapland Central Hospital. C-reactive protein (CRP) level, white blood cell count, leukocyte differentials and arterial blood gas test were analysed using standard methods at the laboratory of Lapland Central Hospital. Pneumonia severity scores were calculated for all patients [20,21].

The treating physician (M.B.) evaluated the possibility of pneumonia among these LRTI patients according to the applicable criteria in terms of patient history, symptoms and physical examination, including systematic chest auscultation, respiratory rate and oxygen saturation. Moreover, CRP levels obtained with the quick test for CRP were available to the physician. Thereafter, the patients visited the study nurse and physician on days 3,7 and 30 .

During these control visits, the study nurse checked the patients' symptoms and the physician checked their clinical condition. The preliminary information on whether the three imaging studies showed opacities compatible with pneumonia was usually available at the first control visit on day 3. Recovery from respiratory infection was confirmed during the control visit on day 30. If initial examination with any of these three methods (i.e. chest radiography, chest MRI or HRCT) showed pulmonary opacities, the examinations were repeated during the follow-up visit to confirm the resolution of the opacities.

\section{Imaging examinations}

All imaging examinations were carried out at the same time. MRI was performed with a 1.5 -T system (Signa HDXt; GE Healthcare, Milwaukee, WI, USA). T2-weighted fast spin echo axial images were obtained with respiratory triggering and an echo train length of $13,320 \times 512$ matrix, effective echo time $\sim 90$ ms and four excitations. Slice thickness $8 \mathrm{~mm}$; gap $1 \mathrm{~mm}$. The imaging time was approximately $3-4 \mathrm{~min}$.

Upright anteroposterior and lateral chest radiographs were obtained with an Aristos VX (Siemens, Erlangen, Germany) imaging system. For HRCT analysis, supine scans using a 64-row multidetector CT scanner were obtained during full inspiration at $10-\mathrm{mm}$ intervals $(1.25 \mathrm{~mm}$ slice thickness, $140 \mathrm{kV}$, automatically modulated mA; LightSpeed VCT; GE Healthcare, Milwaukee, WI, USA). The chest radiography and MRI results were compared with the HRCT results, as HRCT is thus far the most sensitive method for demonstrating pulmonary opacities [16]. During the control visit on day 30, MRI, HRCT and chest radiography were repeated if pneumonia was detected or suspected in the first imaging studies.

All images of the three modalities were analysed for the presence of opacities compatible with pneumonia, whether pneumonia was unilateral or bilateral. The number of affected lobes $(n=1-5)$ was also recorded. Moreover, we evaluated the involvement of lung volume by pneumonic infiltrates $(<20 \%, 21-49 \%, 50-80 \%$ or $>80 \%$ ) as well as the location of infiltrates (peripheral, central and diffuse). 
The criterion for the diagnosis of pneumonia on HRCT was the presence of any interstitial or alveolar ground-glass or reticular opacity or consolidation associated with different types of pneumonia [22]. The MRI examinations were evaluated for increased signal intensity on T2-weighted images appearing as consolidations, patchy nodular lesions or less-intense ground-glass lesions. Digital imaging data were reviewed using a diagnostic workstation (neaView Radiology version 2.23; Neagen, Oulu, Finland) equipped with two diagnostic quality 3-megapixel greyscale monitors. Two experienced radiologists (A.J. and E.P.) analysed the images independently and were blinded to the patients' clinical data. If their initial opinions differed, a consensus was reached.

\section{Statistical analysis}

Statistical analyses were performed using SPSS Statistics for Windows version 21.0 (IBM, Armonk, NY, USA). The summary data are presented as median (interquartile range (IQR)). ANOVA or the Brown-Forsythe test was used with continuous variables because there were more than two groups, the latter test if the p-value according to the homogeneity test of variances was $<0.05$. Ordinal variables were analysed using the KruskalWallis test. The Chi-squared or Fisher exact test was used for categorical variables. Diagnostic differences between HRCT, MRI and chest radiography were compared using the Cochran Q-test; if it showed significant difference $(\mathrm{p}<0.05)$ then pairwise comparisons were performed using the McNemar test (dichotomous variables). Diagnostic differences between ordinal variables were analysed using the Friedman test. The sensitivity, specificity, and positive and negative likelihood ratios with $95 \%$ confidence intervals were calculated for the chest radiography and MRI results using HRCT as a reference. Two-tailed p-values are reported.

\section{Results}

From January 1, 2008 to January 11, 2012, a total of 80 adult LRTI patients participated in this study. Three patients $(3.8 \%)$ were unable to undergo MRI: one due to claustrophobia and two due to profuse coughing. The final study population consisted of 77 ambulatory patients (33 females (42.9\%) and 44 males $(57.1 \%))$ with a median (IQR) age of $43(30-52)$ years.

All three imaging methods were performed at the same time. In our series of 77 adult ambulatory LRTI patients, HRCT identified pneumonia in 32 patients (41.6\%), while MRI showed abnormal signal compatible with pneumonia in 30 patients. Pneumonia was missed in two patients due to motion artefacts. The remaining 45 patients with normal chest MRI were considered to have acute tracheobronchitis. In the follow-up HRCT and MRI examinations each area of opacity had dissolved and none of them proved to be false-positive findings. Compared with the results obtained with HRCT, MRI had a sensitivity of 0.938, specificity of 0.978 , positive likelihood ratio of 42.2 and negative likelihood ratio of 0.06 (table 1).

In the follow-up chest radiography, four out of 27 opacities (14.8\%) initially considered pneumonic proved to be chronic changes: in three cases with bronchiectasis and in one case with fibrotic lesions. Two of these patients had been diagnosed previously with bronchiectasis and one had chronic pulmonary changes after the clinical recovery of sarcoidosis. Compared with the ability of HRCT to detect pneumonic opacities, chest radiography had a sensitivity of 0.719 , specificity of 0.911 , positive likelihood ratio of 8.1 and negative likelihood ratio of 0.31 (table 1). One pneumonia case detected by both chest radiography and HRCT was negative on MRI due to a motion artefact.

When the three imaging methods were compared with regard to their ability to detect the presence of pneumonia and the extent of the involvement of opacities in the lung (unilateral versus bilateral or the number of affected lobes), they differed significantly from each other (table 2). HRCT was the most accurate method and chest MRI was superior to chest radiography (figure 1). In all three modalities, the affected lung volume was $<20 \%$ in more than half of the cases. The location of infiltrates was typically diffuse in each of the three modalities (table 2). According to the three imaging modalities, none of our LRTI patients had parapneumonic effusion or empyema.

TABLE 1 Diagnostic accuracy of chest magnetic resonance imaging (MRI) and chest radiography for identifying pneumonia among 77 adult ambulatory patients with lower respiratory tract infection

MRI

Chest radiography

Sensitivity $(95 \% \mathrm{CI})$

Specificity $(95 \% \mathrm{CI})$

Positive likelihood ratio $(95 \% \mathrm{CI})$

Negative likelihood ratio $(95 \% \mathrm{CI})$
$0.938(0.799-0.983)$
$0.978(0.884-0.996)$
$42.2(6.1-293.6)$
$0.06(0.02-0.25)$

$0.719(0.546-0.844)$

$0.911(0.793-0.965)$

$8.1(3.1-21.1)$

$0.31(0.18-0.54)$

High-resolution computed tomography results were used as reference. 


\begin{tabular}{|c|c|c|c|}
\hline & HRCT & MRI & Chest radiography \\
\hline Pneumonia present ${ }^{\#}$ & $32(41.6)$ & $30(39.0)$ & $23(29.9)$ \\
\hline Unilateral ${ }^{\text {I1 }}$ & 15 (19.5) & $16(20.8)$ & $16(20.8)$ \\
\hline Bilateral ${ }^{\uparrow}$ & $17(22.1)$ & $14(18.2)$ & $7(9.1)$ \\
\hline \multicolumn{4}{|l|}{ Affected lobes $n^{\pi}$} \\
\hline 0 & $45(58.4)$ & $47(61.0)$ & $54(70.1)$ \\
\hline 1 & $11(14.3)$ & 13 (16.9) & $14(18.2)$ \\
\hline 2 & $8(10.4)$ & $11(14.3)$ & $5(6.5)$ \\
\hline 3 & $6(7.8)$ & $2(2.6)$ & $2(2.6)$ \\
\hline 4 & $3(3.9)$ & $3(3.9)$ & $1(1.3)$ \\
\hline 5 & $4(5.2)$ & $1(1.3)$ & $1(1.3)$ \\
\hline \multicolumn{4}{|c|}{ Affected lung volume of opacities \% } \\
\hline$<20$ & $17(53.1)$ & 22 (73.3) & $15(65.2)$ \\
\hline $20-49$ & $15(46.9)$ & $8(26.7)$ & $7(30.4)$ \\
\hline $50-80$ & 0 & 0 & $1(4.3)$ \\
\hline \multicolumn{4}{|l|}{ Location of opacities } \\
\hline Peripheral & $3(9.4)$ & $5(16.7)$ & $2(9.1)$ \\
\hline Central & $2(6.3)$ & $1(3.3)$ & $3(13.6)$ \\
\hline Diffuse & $27(84.4)$ & $24(80.0)$ & $17(77.3)$ \\
\hline
\end{tabular}

\begin{abstract}
A comparison between those LRTI patients who had pulmonary opacities detected by both chest radiography and MRI $(n=22)$ and those 46 patients without any pneumonic changes showed that there were more smokers among patients with pneumonia (table 3). The CURB-65 (confusion, urea, respiratory rate, blood pressure and age $\geqslant 65$ years) severity scores and the Pneumonia Severity Index (PSI) scores did not differ between the patients with and without pneumonia (table 3). Of the 77 LRTI patients, 66 (85.7\%) belonged to class 0 according to the CURB-65 severity score and 58 (75.3\%) to class I according to the PSI score (table 3). Patients with pneumonia detected by both MRI and chest radiography $(\mathrm{n}=22)$ had the statistically lowest oxygen saturation values and highest CRP concentrations (table 4).

On the basis of data available during the first visit, the physician started antibiotics for possible pneumonia in 40 out of 77 patients (51.9\%). He initiated three antibiotic courses on the basis of the later information from the imaging results. Antibiotics were prescribed most often for patients who in later chest radiography and MRI examinations were shown to have pulmonary opacities compatible with pneumonia (19 out of 22 patients $(86.4 \%))$ (table 4$)$.
\end{abstract}
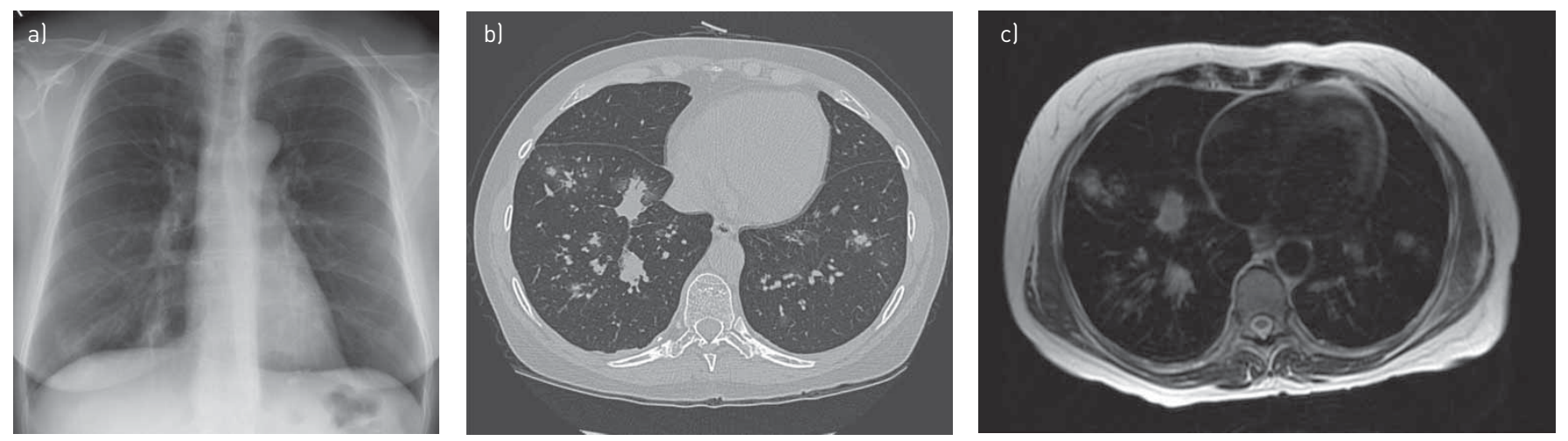

FIGURE 1 Imaging revealed consolidations in a 55-year-old tobacco-smoking woman: a) chest radiography revealed a pneumonic consolidation in the right lower lobe, b) high-resolution computed tomography (HRCT) revealed bilateral pneumonic consolidations with some ground-glass opacities and c) T2-weighted magnetic resonance imaging revealed bilateral consolidations comparable to the HRCT findings. The patient sought help after experiencing respiratory symptoms (i.e. productive cough and chest pain) and fever with a peak temperature of $38.2^{\circ} \mathrm{C}$ for 6 days. She had a C-reactive protein level of $12.9 \mathrm{mg} \cdot \mathrm{dL}^{-1}$, white blood cell count of $12.9 \times 10^{9} \mathrm{~L}^{-1}$, heart rate of 106 beats $\mathrm{min}^{-1}$, respiratory rate of 16 breaths $\mathrm{min}^{-1}, 93 \%$ oxygen saturation and bilateral crackles on chest auscultation. 
TABLE 3 Characteristics and pneumonia severity scores of 77 ambulatory patients with lower respiratory tract infection with $(+)$ and without $(-)$ pneumonia as detected by chest magnetic resonance imaging (MRI) examination or chest radiography (CR)

\begin{tabular}{|c|c|c|c|c|c|c|}
\hline & All & MRI-/CR- & MRI+/CR- & $\mathrm{MRI}+/ \mathrm{CR}+$ & MRI-/CR+ & p-value ${ }^{\#}$ \\
\hline Subjects & 77 & 46 & 8 & 22 & 1 & \\
\hline Age years & $43(30-52)$ & $41.5(30-51)$ & $38(23.5-56.5)$ & $45(35-54)$ & 36 & 0.79 \\
\hline Female & $33(42.9)$ & 19 (41.3) & 5 (62.5) & $9(40.9)$ & 0 & 0.58 \\
\hline Underlying diseases & $26(33.8)$ & $17(37.0)$ & $4(50.0)$ & $5(22.7)$ & 0 & 0.30 \\
\hline Smoking & $33(42.9)$ & $18(39.1)$ & 1 (12.5) & $14(63.6)$ & 0 & 0.036 \\
\hline Alcohol abuse & $9(11.7)$ & $6(13.0)$ & 0 & $3(13.6)$ & 0 & 0.76 \\
\hline Symptom duration days & $5(4-6)$ & $5(3-6)$ & $4.5(3.5-6)$ & $5(4-7)$ & 6 & 0.79 \\
\hline Productive cough & $69(89.6)$ & $41(89.1)$ & $8(100)$ & $19(86.4)$ & 1 & 0.75 \\
\hline Chest pain & $38(49.4)$ & 20 (43.5) & $4(50.0)$ & $13(59.1)$ & 1 & 0.52 \\
\hline CURB-65 score & & & & & & 0.29 \\
\hline 0 & 66 (85.7) & $38(82.6)$ & $6(75.0)$ & 21 (95.5) & 1 & \\
\hline 1 & $8(10.4)$ & $7(15.2)$ & $1(12.5)$ & 0 & & \\
\hline 2 & $3(3.9)$ & $1(2.2)$ & 1 (12.5) & $1(4.5)$ & & \\
\hline PSI score & & & & & & 0.82 \\
\hline I & 58 (75.3) & $35(76.1)$ & $5(62.5)$ & 17 (77.3) & 1 & \\
\hline ॥ & 15 (19.5) & 8 (17.4) & 3 (37.5) & 4 (18.2) & & \\
\hline III & $2(2.6)$ & $2(4.3)$ & 0 & 0 & & \\
\hline IV & $2(2.6)$ & $1(2.2)$ & 0 & $1(4.5)$ & & \\
\hline
\end{tabular}

Data are presented as $\mathrm{n}$, median (interquartile range) or $\mathrm{n}(\%)$, unless otherwise stated. CURB-65: confusion, urea, respiratory rate, blood pressure and age $\geqslant 65$ years [20]; PSI: Pneumonia Severity Index [21]. ${ }^{\#}$ : MRI-/CR+ group omitted from statistical analysis due to the small number of cases $(n=1)$.

\section{Discussion}

Our results showed that chest MRI examination was well tolerated and a single T2-weighted respiratory-triggered series with an imaging time of a few minutes produced excellent results. Among 77 adult ambulatory LRTI patients, MRI missed only two of the 32 pneumonia cases that showed positive in HRCT and this was due to motion artefacts. In addition, MRI was significantly better than chest radiography in detecting opacities compatible with pneumonia (30 versus 23 detected cases).

One crucial limitation for the use of MRI for lung imaging is the examination time. We chose to use only a single respiratory-triggered fast spin echo T2-weighted sequence, which proved sufficient for detecting

TABLE 4 Physical and laboratory findings and prescription of antibiotics in 77 ambulatory patients with lower respiratory tract infection with (+) and without (-) pneumonia as detected by chest magnetic resonance imaging (MRI) examination or chest radiography $(\mathrm{CR})$

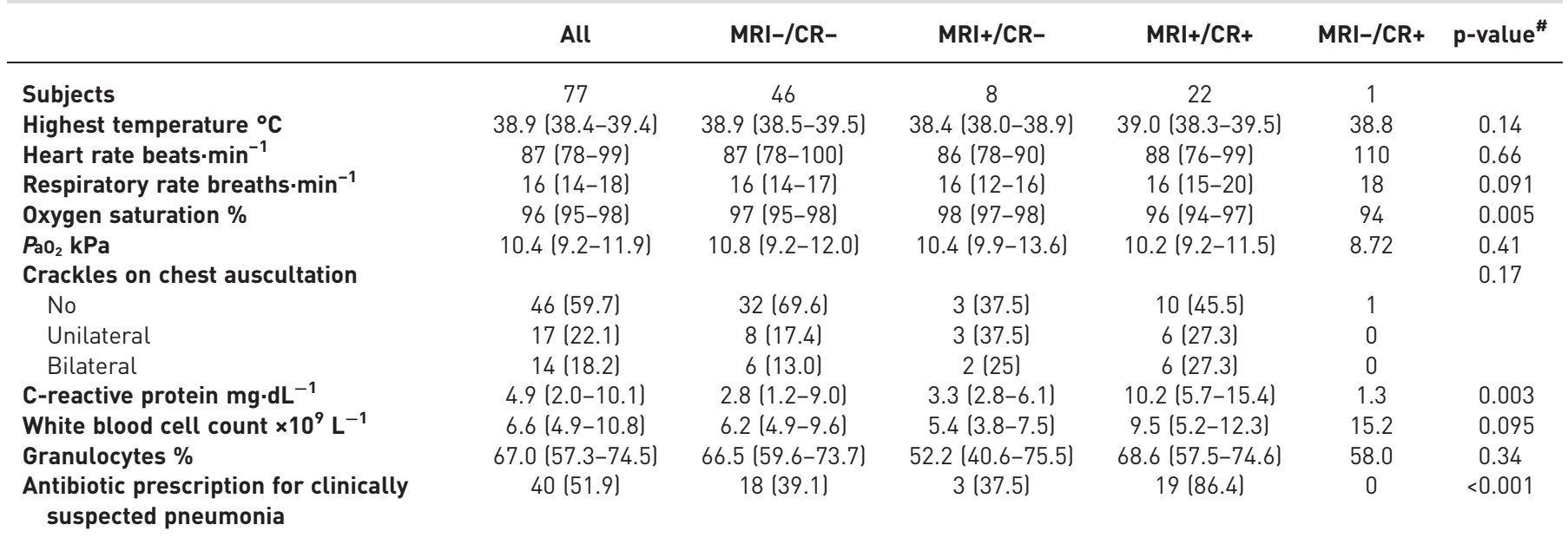

Data are presented as $\mathrm{n}$, median (interquartile range) or $\mathrm{n}(\%)$, unless otherwise stated. $\mathrm{PaO}_{2}$ : arterial oxygen tension. ${ }^{\#}: \mathrm{MRI}-/ \mathrm{CR}+\mathrm{group}$ omitted from statistical analysis due to the small number of cases $(n=1)$. 
pneumonia. The imaging time was only 3-4 min, with only three patients failing the examination. T2-weighted sequences are sensitive to water accumulation with high proton density. Pneumonic infiltrates and nodules have high proton density and appear as areas of high signal intensity in the background of low-intensity aerated normal lung tissue. Taken together, our results suggest that relevant clinical information can be obtained with a single sequence with a short imaging time when pneumonia is suspected in ambulatory patients. According to our 1-month follow-up results, pulmonary opacities detected by MRI disappeared in a manner similar to that detected during HRCT and chest radiography. The financial cost of chest MRI may limit the wider use of this method. In our institution, the standard cost of MRI with up to four sequences is almost nine times higher than that of chest radiography. If only one sequence would be used in clinical situations, as we did in this study, the cost could be reduced by half; it would then be approximately four times higher than that of chest radiography.

As far as we are aware, two previous studies have compared chest MRI (1.5 T) and chest CT examinations $[8,9]$. Both studies included immunosuppressive patients whose chest radiography did not reveal opacities compatible with pneumonia. In the first study, among 30 studied patients thin-section helical CT identified 22 cases of pneumonia (73\%), while MRI found 21 of these pneumonia cases (95\%) and produced one false-positive finding among the eight cases negative in CT; this gave a sensitivity of 0.95 and specificity of 0.88 for chest MRI [8]. In the second study, MRI was performed in 33 HRCT-identified pneumonia cases, of which MRI found 30 cases (91\%). In our series of 77 adult ambulatory LRTI patients, HRCT identified pneumonia in 32 patients; in 30 of them (94\%) pneumonia was also observed by chest MRI. Two cases were missed due to motion artefacts. In our series we did not observe any false-positive results in MRI imaging. Taken together, chest MRI is almost as good as HRCT for the identification of pneumonia cases among ambulatory LRTI patients.

When the results of MRI and chest radiography were compared, chest radiography missed almost 30\% of the pneumonia cases identified by MRI, and it also produced false-positive results with poorer sensitivity (0.94 versus 0.72 ) and specificity (0.98 versus 0.91$)$. Chest MRI is a promising method for diagnosing pneumonia when ionising radiation may be harmful. Based on likelihood ratios, MRI was an excellent method for diagnosing pneumonia in our LRTI series; the positive likelihood ratio of 42.2 indicates that opacities identified by chest MRI during acute respiratory infection are usually due to pneumonia and a negative result (negative likelihood ratio 0.06 ) practically rules out the possibility of pneumonia [23].

Our prospective study has several strengths. To the best of our knowledge, our study is the first to assess the use of MRI in diagnosing CAP in adult ambulatory patients with LRTI. All patients were examined and treated by the same physician specialised in infectious diseases. Moreover, MRI was compared with simultaneously obtained HRCT and chest radiography results with follow-up verification of the resolution of the opacities if detected in the first examination, with two experienced radiologists interpreting all imaging findings independently. Our results concerning HRCT and chest radiography are in agreement with a recent multicentre study [19] in which chest radiography also resulted in both false-negative and false-positive findings.

However, our study does have some limitations. The study period for such a common disease was quite long. The delay was due to pandemic influenza and the fact that our study nurse was not at our disposal for a period of 8 months. This was also a single-centre study with a rather small number of ambulatory LRTI patients, which limits the generalisability of the results, especially to more severe cases of respiratory infections. However, the above-mentioned studies that compared chest CT and MRI as methods for diagnosing pneumonia in immunosuppressive neutropenic patients showed practically similar results.

In conclusion, MRI is an alternative to chest radiography, and even to CT, for diagnosing pneumonia when ionising radiation may be harmful (e.g. in paediatric, young or pregnant patients). In addition, it has been previously reported to be a promising technique among immunosuppressive patients whose chest radiography did not reveal opacities compatible with pneumonia $[8,9]$. Our results encourage the broader use of fast and well-tolerated chest MRI for also diagnosing CAP among adults. Accurate chest MRI examinations would be preferred when repeated examinations are considered clinically relevant, especially in the above-mentioned patient groups.

\section{Acknowledgements}

We thank study nurse Arja Kähkölä (Lapland Central Hospital, Rovaniemi, Finland) for her excellent work.

Author contributions: H. Syrjala and M. Broas conceived the study. H. Syrjala, M. Broas, P. Ohtonen, A. Jartti and E. Pääkkö designed the study. H. Syrjala and P. Ohtonen performed the analysis. H. Syrjala, M. Broas, P. Ohtonen, A. Jartti and E. Pääkkö interpreted the results. H. Syrjala wrote the initial draft of the manuscript. H. Syrjala, M. Broas, P. Ohtonen, A. Jartti and E. Pääkkö critically revised the manuscript and approved the final version.

\section{References}

1 Mandell LA, Wunderink RG, Anzueto A, et al. Infectious Diseases Society of America/American Thoracic Society consensus guidelines on the management of community-acquired pneumonia in adults. Clin Infect Dis 2007; 44: S27-S72. 
2 Lim WS, Baudouin SV, George RC, et al. British Thoracic Society guidelines for the management of community acquired pneumonia in adults: update 2009. Thorax 2009; 64: Suppl. III, 1-55.

3 Woodhead M, Blasi F, Ewig S, et al. Guidelines for the management of adult lower respiratory tract infections. Clin Microbiol Infect 2011; 17: Suppl. 6, E1-E59.

4 Wagner M, Böwing BC, Kuth R, et al. Low field thoracic MRI - a fast and radiation free routine imaging modality in children. Magn Reson Imaging 2001; 19: 975-983.

5 Peltola V, Ruuskanen O, Svedström E. Magnetic resonance imaging of lung infections in children. Pediatr Radiol 2008; 38: 1225-1231.

6 Yikilmaz A, Koc A, Coskun A, et al. Evaluation of pneumonia in children: comparison of MRI with fast imaging sequences at $1.5 \mathrm{~T}$ with chest radiographs. Acta Radiol 2011; 52: 914-919.

7 Hirsch W, Sorge I, Krohmer S, et al. MRI of the lungs in children. Eur J Radiol 2008; 68: 278-288.

8 Eibel R, Herzog P, Dietrich O, et al. Pulmonary abnormalities in immunocompromised patients: comparative detection with parallel acquisition MR imaging and thin-section helical CT. Radiology 2006; 241: 880-891.

9 Rieger C, Herzog P, Eibel R, et al. Pulmonary MRI - a new approach for the evaluation of febrile neutropenic patients with malignancies. Support Care Cancer 2008; 16: 599-606.

10 Attenberger UI, Morelli JN, Henzler T, et al. 3 Tesla proton MRI for the diagnosis of pneumonia/lung infiltrates in neutropenic patients with acute myeloid leukemia: initial results in comparison to HRCT. Eur J Radiol 2014; 83: e61-e66.

11 Wielpuz M, Kauczor HU. MRI of the lung: state of the art. Diagn Interv Radiol 2012; 18: 344-353.

12 Wild JM, Marshall H, Bock M, et al. MRI of the lung (1/3): methods. Insights Imaging 2012; 3: 345-353.

13 Biederer J, Beer M, Hirsch W, et al. MRI of the lung (2/3). Why ... when ... how? Insights Imaging 2012; 3: 355-371.

14 Biederer J, Mirsadraee S, Beer M, et al. MRI of the lung (3/3) - current applications and future perspectives. Insights Imaging 2012; 3: 373-386.

15 Montella S, Santamaria F, Salvatore M, et al. Assessment of chest high-field magnetic resonance imaging in children and young adults with noncystic fibrosis chronic lung disease: comparison to high-resolution computed tomography and correlation with pulmonary function. Invest Radiol 2009; 44: 532-538.

16 Webb WR, Muller NL, Naidich DP. High-resolution computed tomography findings of lung diseases. In: Webb WR, Muller NL, Naidich DP. High Resolution CT of the Lung. 5th Edn. Philadelphia, Wolters Kluwer Health, 2015; pp. 429-480.

17 Syrjälä H, Broas M, Suramo I, et al. High-resolution computed tomography for the diagnosis of community-acquired pneumonia. Clin Infect Dis 1998; 27: 358-363.

18 Self WH, Courtney DM, McNaughton CD, et al. High discordance of chest x-ray and computed tomography for detection of pulmonary opacities in ED patients: implications for diagnosing pneumonia. Am J Emerg Med 2013; 31: 401-405.

19 Claessens YE, Debray MP, Tubach F, et al. Early chest CT-scan to assist diagnosis and guide treatment decision for suspected community-acquired pneumonia. Am J Respir Crit Care Med 2015; 450: 97-103.

20 Lim WS, van der Eerden MM, Laing R, et al. Defining community-acquired pneumonia severity on presentation to hospital: an international derivation and validation study. Thorax 2003; 58: 377-382.

21 Fine MJ, Auble TE, Yealy DM, et al. A prediction rule to identify low-risk patients with community-acquired pneumonia. N Engl J Med 1997; 336: 243-250.

22 Hansell DM, Lynch DA, Mc Adams HP, et al. Basic HRCT patterns of lung disease. In: Hansell DM, Lynch DA, McAdams HP, et al., eds. Imaging of Diseases of the Chest. 5th Edn. St Louis, Mosby Elsevier, 2010; pp. 153-204.

23 Jaeschke R, Guyatt GH, Sackett DL. Users' guides to the medical literature. III. How to use an article about a diagnostic test. B. What are the results and will they help me in caring for my patients? The Evidence-Based Medicine Working Group. JAMA 1994; 271: 703-707. 